\title{
Analysis of Coupled Gas Flow and Solid Deformation in Porous Media
}

\author{
Lei Su, ${ }^{1, a}$, Liang $\mathrm{Mi}^{2, \mathrm{~b}}$ and $\mathrm{Xi}$ Chen ${ }^{3, \mathrm{c}}$ \\ 1,2 China Merchants Chongqing Communications Technology Research \& Design Institute CO.,LTD, \\ Chongqing 400067, China \\ ${ }^{3}$ Sichuan University of Science \& Engineering, Zigong 643000, China \\ asl_n@163.com, ${ }^{b}$ oilmar@126.com, ${ }^{c} 123 c x @$ tongji.edu.cn
}

Keywords: coupled problem, Klinkenberg effects, analytical method, Boltzmann transformation.

Abstract. The coupled problem between gas flow and solid deformation in porous media analysis has important influence in contaminant transport and gas outbursts during coal mining. In this paper, it is presented an analytical method to solve coupled nonlinear models of stress and gas seepage, which consider the Klinkenberg effects as gas penetrating into porous medium. The method is based on integrating the stress model and changed the coupled equations into an nonlinear ordinary differential equation by Boltzmann variable, then an approximation is constructed for simulation. At last, an illustration is given to confirm the accuracy of present solution.

\section{Introduction}

In present study, the gas migration in coal or rock seam is a coupled process between the gas flow and solid mechanical deformation ${ }^{[1]}$. The process is defined by coupled equations of seepage and stress, which are composed by gas flow equation and porous medium mass deformation one. The gas flow is different from liquid flow because of its effective permeability effect. According to Sun, the gas infiltration considered the Klinkenberg effects can be expressed as ${ }^{[1]}$

$2 \alpha P \frac{\partial \varepsilon_{v}}{\partial t}+\left[2 \varphi+\frac{2(1-\varphi)}{k_{s}} P\right] \frac{\partial P}{\partial t}=\frac{\partial}{\partial x}\left(\frac{k_{\infty}}{\mu_{g}}\left(1+\frac{b}{P}\right) \frac{\partial P^{2}}{\partial x}\right)$,

where is Biot's effective stress parameter, $P$ is gas pore pressure $\left[\mathrm{L}^{-1} \mathrm{MT}^{-2}\right], x$ and $t$ are the space and the time coordinates, $\quad v(=\partial u / \partial x)$ is volume strain [-], $u$ is displacement [L] in porous medium, is porosity [-], $k_{s}$ is bulk modulus of solid grains $\left[\mathrm{L}^{-1} \mathrm{MT}^{-2}\right], k_{\infty}$ is absolute gas permeability under very large gas pressure $\left[\mathrm{L}^{2}\right], \quad g$ is gas dynamic viscosity coefficient $\left[\mathrm{L}^{-1} \mathrm{MT}^{-1}\right], b$ is Klinkenberg factor $\left[\mathrm{L}^{-1} \mathrm{MT}^{-2}\right]$. The mass deformation of porous medium is

$E \frac{\partial \varepsilon_{v}}{\partial x}-\alpha \frac{\partial P}{\partial x}+F=0$

in which $E$ is Young's modulus $\left[\mathrm{L}^{-1} \mathrm{MT}^{-2}\right]$ and $F$ is body force $\left[\mathrm{L}^{-2} \mathrm{MT}^{-2}\right]$. In this paper, our goal is analyzing the coupled Eqs. (1)-(2) and obtaining an analytical method to approximate their solutions.

Nowadays, many approaches, for instance, Heaslet \& Alksne technique ${ }^{[2]}$, series expansion method $^{[3]}$ and traveling wave method ${ }^{[4]}$ are used to obtain the analytical solution of flow equation (1). Inverse method and semi-inverse method ${ }^{[5]}$ are applied for some linear stress equation (2). Nevertheless, there are not too many analytical solutions in non-linear fully-coupled seepage and stress. Influenced by the researches before, it is integrated the stress model and changed the coupled equations (1)-(2) into a diffusion partial differential equation (PDE), which could be transformed into an ordinary differential equation (ODE) by Boltzmann variable. Then an intermediate variable is constructed to obtain the approximate solution of the diffusion PDE. An example is given to test the present solution.

\section{Initial and Boundary Conditions}

It is considered a semi-infinite boundary conditions as 


$$
\left\{\begin{array}{l}
\left.P\right|_{x=0}=P_{L} \\
\left.P\right|_{x \rightarrow+\infty}=P_{0}
\end{array},\left\{\begin{array}{l}
\left.u\right|_{x=0}=u_{L} \\
\left.u\right|_{x \rightarrow+\infty}=0
\end{array},\right.\right.
$$

in which $P_{L}, P_{0}$ and $u_{L}$ are known constants. The initial conditions are

$$
\left.P\right|_{t=0}=P_{0},\left.u\right|_{t=0}=0 \text {. }
$$

\section{Solving the Coupled Equations}

If body force $F$ is neglected, the stress model in (2) can be integrated based on $t=0$ time

$E \varepsilon_{v}=\alpha P-\alpha P_{0}$,

Substituting (5) into the gas flow model in (1), it is obtained

$$
\frac{\partial U}{\partial t}=\frac{\partial}{\partial x}\left(\frac{k_{\infty}}{c \mu_{g}}\left(\frac{b+P}{c_{1}+P}\right) \frac{\partial U}{\partial x}\right)
$$

in which

$$
c=\frac{\alpha^{2}}{E}+\frac{(1-\varphi)}{k_{s}}, c_{1}=\frac{\varphi}{c}, U=\frac{1}{2}\left(\frac{\varphi}{c}+P\right)^{2}-\frac{1}{2}\left(\frac{\varphi}{c}+P_{0}\right)^{2} .
$$

From Eq. (7), the initial and boundary conditions for fluid flow process (6) are changed into $\left.U\right|_{x=0}=U_{L},\left.U\right|_{x \rightarrow+\infty}=0,\left.U\right|_{t=0}=0$,

where $U_{L}$ is

$$
U_{L}=\frac{1}{2}\left(\frac{\varphi}{c}+P_{L}\right)^{2}-\frac{1}{2}\left(\frac{\varphi}{c}+P_{0}\right)^{2} \text {. }
$$

According to Parlange ${ }^{[6]}$, Eq. (6) with Eq. (7) can be shown as

$$
\frac{\mathrm{d} \phi}{\mathrm{d} U} \int_{0}^{U} \phi \mathrm{d} U=-2 D(U),
$$

where $D(U)$ is

$$
D(U)=\frac{k_{\infty}}{c \mu_{g}}\left(\frac{\sqrt{2 U+\left(\frac{\varphi}{c}+P_{0}\right)^{2}}-c_{1}+b}{\sqrt{2 U+\left(\frac{\varphi}{c}+P_{0}\right)^{2}}}\right),
$$

and the initial-boundary conditions Eq. (8) are ${ }^{[6]}$

$\left.\phi\right|_{U=U_{L}}=0,\left.\phi\right|_{U=0} \rightarrow+\infty$.

It is constructed $n_{1}(=1,2,3, \ldots)$ order approximate solution of $\quad$ in Eq. (10) is ${ }^{[7]}$

$\phi \approx \sum_{i=1}^{n_{1}} V_{i}\left(\int_{U}^{U_{L}} \frac{D(U)}{U} \mathrm{~d} U\right)^{i}$.

Applying collocation method to Eq. (10), $V_{i}$ can be calculated. According to Eq. (5) and Eq. (7), the volume strain $\quad v$ is

$\varepsilon_{v}=\frac{\alpha}{E}\left[\sqrt{2 U+\left(\frac{\varphi}{c}+P_{0}\right)^{2}}-\frac{\varphi}{c}\right]-\frac{\alpha}{E} P_{0}$,

and displacement $u$ can be shown as

$u=\int_{0}^{x} \varepsilon_{v} \mathrm{~d} x+u_{L}$ 


\section{Numerical Simulation}

In this section, an examples for gas migration in rock seam is given to confirm the accuracy of present method.

Example $E=2.45 \times 10^{10} \mathrm{~Pa}$,

$g=1.84 \times 10^{-5} \mathrm{~Pa} \cdot \mathrm{s}, b=7.6 \times 10^{5} \mathrm{~Pa}, P_{0}=1.01 \times 10^{5} \mathrm{~Pa}, P_{L}=2 \times 10^{5} \mathrm{~Pa}, u_{L}=0.002 \mathrm{~m}$.

According to (9) and (13), $U_{L}$ is $1.48996 \times 10^{10} \mathrm{~Pa}$, and we obtain

$$
\frac{x}{\sqrt{t}} \approx \sum_{i=1}^{n_{1}} V_{i}\left(\int_{U}^{U_{L}} \frac{1.854 \times 10^{-13}\left(\frac{\sqrt{2 U+1.02012 \times 10^{10}}+7.5999 \times 10^{5}}{\sqrt{2 U+1.02012 \times 10^{10}}}\right)}{U} \mathrm{~d} U\right)^{i} .
$$

The calculation parameter $V_{i}$ and collocation position $U_{k}(k=1,2,3, . ., 6)$ of $1-6$ order approximation in (16) are

$$
\begin{aligned}
& n_{1}=1: V_{1}=2.475721117 \times 10^{-7}, U_{1}=U_{L} . \\
& n_{1}=2: V_{1}=2.941147935 \times 10^{-7}, V_{2}=-6.035403844 \times 10^{-9}, U_{1}=U_{L}, U_{2}=0.1 U_{L} . \\
& n_{1}=3: V_{1}=3.019271928 \times 10^{-7}, V_{2}=-1.936508206 \times 10^{-8}, V_{3}=5.312412542 \times 10^{-10}, U_{1}=U_{L}, U_{2}=0.1 U_{L}, \\
& U_{3}=0.01 U_{L} . \\
& n_{1}=4: V_{1}=3.176300790 \times 10^{-7}, V_{2}=-2.063319029 \times 10^{-8}, V_{3}=7.465580921 \times 10^{-10}, \\
& V_{4}=-7.317388182 \times 10^{-12}, U_{1}=U_{L}, U_{2}=0.1 U_{L}, U_{3}=0.05 U_{L}, U_{4}=0.001 U_{L} . \\
& n_{1}=5: V_{1}=3.215204622 \times 10^{-7}, V_{2}=-2.202903455 \times 10^{-8}, V_{3}=1.056141767 \times 10^{-9}, \\
& V_{4}=-2.241773151 \times 10^{-11}, V_{5}=1.645978533 \times 10^{-13}, U_{1}=U_{L}, U_{2}=0.1 U_{L}, U_{3}=0.5 U_{L}, U_{4}=0.05 U_{L}, \\
& U_{5}=0.005 U_{L} . \\
& n_{1}=6: V_{1}=3.222305293 \times 10^{-7}, V_{2}=-2.372596833 \times 10^{-8}, V_{3}=1.478140057 \times 10^{-9}, \\
& V_{4}=-5.037007960 \times 10^{-11}, V_{5}=8.246836742 \times 10^{-13}, V_{6}=-4.924314338 \times 10^{-15}, U_{1}=U_{L}, U_{2}=0.1 U_{L}, \\
& U_{3}=0.5 U_{L}, U_{4}=0.05 U_{L}, U_{5}=0.005 U_{L}, U_{6}=0.001 U_{L} .
\end{aligned}
$$

Comparing with finite element method (FEM) and calculating for results with pressure $P$ calculated by Eq. (7) in $t=10000$ s as the exact solution, the 1-6 order approximate solutions of $P$ are shown in Fig. 1. The results of $P$ obtained from 6 order approximation are compared with FEM in Table 1.

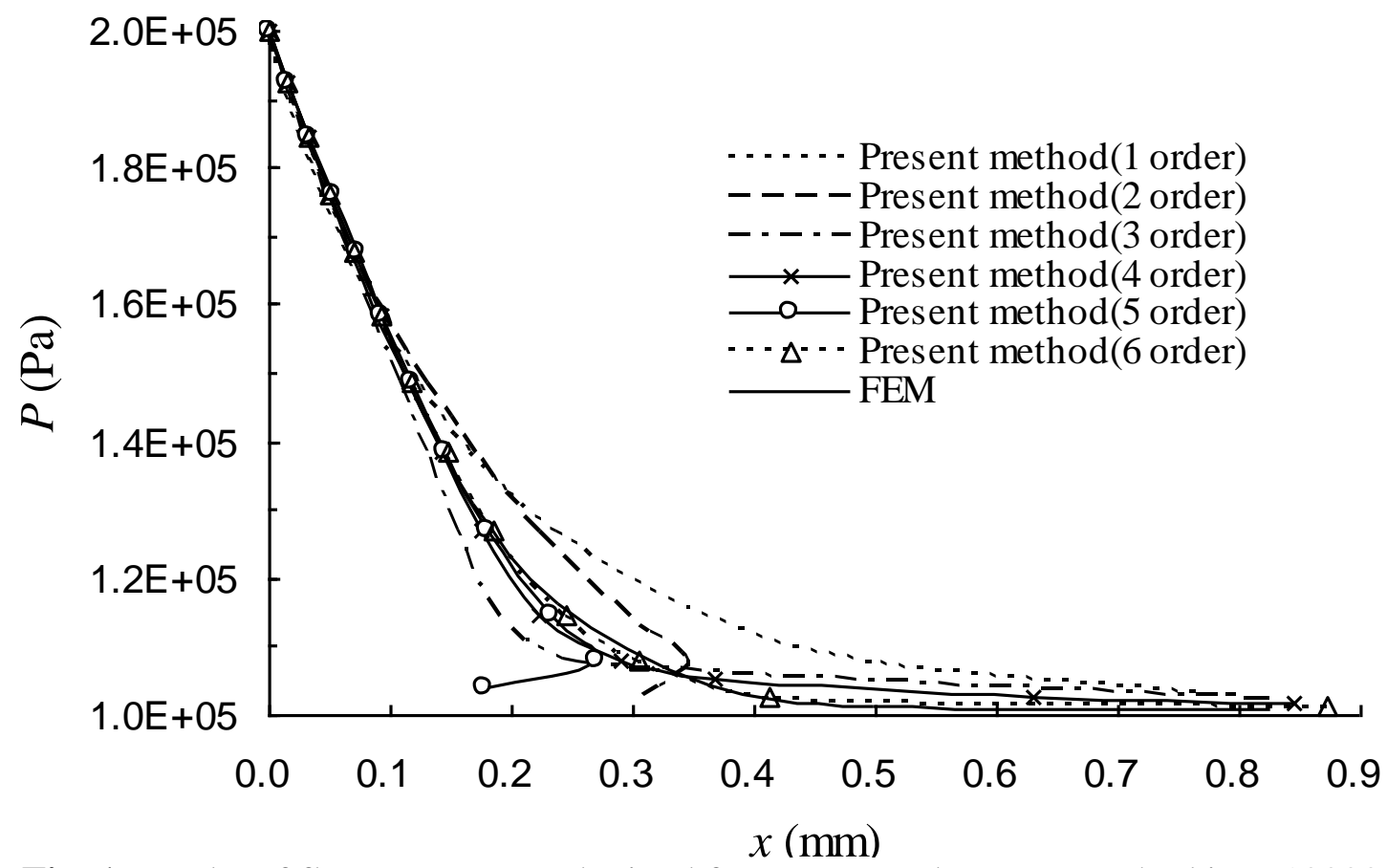

Fig. 1 Results of flow pressure $P$ obtained from FEM and present method in $t=10000 \mathrm{~s}$ 
Table 1: Results of $P$ obtained from FEM and present method ( $t=10000 \mathrm{~s})$

\begin{tabular}{cccc}
\hline$x(\mathrm{~mm})$ & 6 order approximate solution $(\mathrm{Pa})$ & FEM $(\mathrm{Pa})$ & Relative error \\
\hline 0.8736 & 101147.412 & 101000 & $0.15 \%$ \\
0.4125 & 102464.574 & 102758.121 & $-0.29 \%$ \\
0.3050 & 108124.675 & 108100.363 & $0.02 \%$ \\
0.2460 & 114808.065 & 114830.120 & $-0.02 \%$ \\
0.1843 & 127125.082 & 126760.857 & $0.29 \%$ \\
0.1465 & 138349.859 & 137257.588 & $0.80 \%$ \\
0.1174 & 148729.897 & 147141.441 & $1.08 \%$ \\
0.0928 & 158431.318 & 156718.095 & $1.09 \%$ \\
0.0710 & 167572.025 & 166051.353 & $0.92 \%$ \\
0.0512 & 176239.286 & 175109.751 & $0.65 \%$ \\
0.0329 & 184499.835 & 183830.398 & $0.36 \%$ \\
0.0159 & 192406.062 & 192146.473 & $0.14 \%$ \\
0 & 200000 & 200000 & $0.00 \%$ \\
\hline
\end{tabular}

In Fig. 1 , it can be obtained that the pressure $P$ decreases from $2 \times 10^{5} \mathrm{~Pa}$ to $1.01 \times 10^{5} \mathrm{~Pa}$ as the space coordinate $x$ increases. The 6 order approximate solution is close to the exact solutions. In Table 1, the maximum relative error value of present method is $1.09 \%$ in $x=0.0928 \mathrm{~mm}$ for 6 order approximation solution.

According to Eq. (14), $v$ is $\varepsilon_{v}=3.459 \times 10^{-6} P-0.3494$.

From Eq. (15), the displacement of $u$ is

$u=\int_{0}^{x} 3.459 \times 10^{-6} P-0.3494 \mathrm{~d} x+0.002$

in which the integration in $u$ is close to 0 . Thus, the displacement can be approximated as $u \approx 0.002$,

which is close to the results from $\operatorname{FEM}(u=0.00201)$.

\section{Conclusion}

In this paper, it is derived the one-dimensional approximation solution of coupled equations of gas flow and solid deformation in porous medium. It is obtained the fully-coupled models Eqs. (1)-(2) can be changed into a diffusion equation by integrating the stress model. Then the Boltzmann variable is introduced and an approximation is constructed for approximating the diffusion equation. The presented example shows the accuracy of the present method by comparing with FEM.

\section{References}

[1] P.D. Sun, D.Q. Yang and B.Y. Chen: Introduction to Coupling Models for Multiphysics and Numerical Simulations, (China Science \& Technology, Beijing 2007).

[2] D. Lockington, J.Y. Parlange and P. Dux: Mater. Struct. Vol. 32 (1999), p. 342

[3] M. Omidvar, A. Barari, M. Momeni and D.D. Ganji: Geomech. Geoeng. Int. J. Vol. 5 (2010), p. 127

[4] T.P. Witelski: Adv. Water Resour. Vol. 28 (2005), p. 1133

[5] Z.L. Xu: Elasticity, (China Higher Education Press, Beijing 2016).

[6] M.B. Parlange, S.N. Prasad, J.Y. Parlange and M.J.M. Romkens: Water Resour. Res. Vol. 28 (1992), p. 2793

[7] X. Chen, Y. Dai: Appl. Math. Mech. Vol. 37 (2016), p. 169 